\title{
Effect of the Earth's oblateness, the Shadow of the Earth due to the Solar Radiation Pressure and Magnetic Force on the Motion and Stability of two satellites connected by an extensible cable in circular orbit of the Centre of Mass.
}

\author{
Vijay Kumar ${ }^{1}$, Umesh Chandra Singh ${ }^{2}$, Nikky Kumari ${ }^{3}$ \\ ${ }^{I}$ (Dept. of Mathematics, Shobhit University, Gangoh, Saharanpur,India) \\ 2 (Dept. of Mathematics, B. R. A. Bihar University, Muzaffarpur, India) \\ ${ }^{3}$ (Research Scholar, Dept. of Mathematics, Dayalbagh Educational Institute, Agra, India)
}

\begin{abstract}
In a linear motion of a system of two satellites connected by extensible cable, one stable equilibrium point exists when perturbative forces like Solar radiation pressure, shadow of the earth, oblateness of the earth, air resistance and earth's magnetic force act simultaneously. We have obtained one stable point of equilibrium in case of perturbative forces like the shadow of the earth due to solar radiation pressure, Magnetic Force and oblateness of the earth acting together on the motion of a system of two satellites connected by extensible cable in the central gravitational field of earth in case of circular orbit of the centre of mass. We have used Liapunov's theorem on stability to examine the stability of the equilibrium point.
\end{abstract}

Key words: Stabiity, Equiliribium Point, Solar Radiation Pressure, circular orbit, Liapunov Theorem, Satellites.

\section{Introduction}

The present paper is concerned with the stability of the equilibrium point of the centre of mass of a system of two satellites connected by a light, flexible and extensible cable under the influence of the shadow of the earth due to solar radiation pressure and oblateness of the earth in case of circular orbit.

Beletsky, V.V is the pioneer worker in this field. This paper is an attempt towards the generalisation of works done by him.

\section{Equations of motion}

The equitation of motion of one of two satellites moving along Keplerian elliptic orbit in Nechvill's coordinates system may be obtained by using Lagrange's equations of motion of first kind in the form:

$$
\begin{aligned}
& x^{\prime \prime}-2 y^{\prime}-3 x \rho-\frac{4 B x}{\rho}+A \rho^{3} \psi \cos \in \cos (v-\alpha)=-\bar{\lambda}_{\alpha} \rho^{4}\left[1-\frac{\ell_{0}}{\rho \sqrt{x^{2}+y^{2}+z^{2}}}\right] x-\frac{C}{\rho} \cos i \\
& \text { and } y^{\prime \prime}+2 x^{\prime}+\frac{B}{\rho} y+A \rho^{3} \psi \cos \in \sin (v-\alpha)=-\bar{\lambda}_{\alpha} \rho^{4}\left[1-\frac{\ell_{0}}{\rho \sqrt{x^{2}+y^{2}+z^{2}}}\right] y-C \frac{\rho^{\prime}}{\rho^{2}} \cos i
\end{aligned}
$$

$$
\begin{aligned}
z^{\prime \prime}+z+\frac{B z}{\rho}-A \rho^{3} \psi \sin & \in=-\bar{\lambda}_{\alpha} \rho^{4}\left[1-\frac{\ell_{0}}{\rho \sqrt{x^{2}+y^{2}+z^{2}}}\right] z- \\
\frac{C}{\rho} \frac{1}{\mu_{E}} & {\left[\frac{\rho^{\prime}}{\rho} \cos (v+w)+\left(3 p^{3} \rho^{3}-\mu_{E}\right) \sin (v+w)\right] \sin i }
\end{aligned}
$$

Where, $\rho=\frac{1}{1+e \cos v} ; r=\sqrt{x^{2}+y^{2}+z^{2}}$

$v=$ True anomaly of the centre of mass of the system

$A=\frac{P^{3}}{\mu}\left(\frac{B_{1}}{m_{1}}-\frac{B_{2}}{m_{2}}\right)=$ Solar radiation pressure parameter 
$B=\frac{3 k_{2}}{P^{2}}=$ Earth's oblateness force parameter.

$C=\left(\frac{Q_{1}}{m_{1}}-\frac{Q_{2}}{m_{2}}\right) \sqrt{\frac{\mu_{E}}{\mu p}}=$ Earth's Magnetic Force Parameters

$\Psi_{1}=$ Earth's shadow function parameter.

$\overline{\lambda_{\alpha}}=\frac{P^{3}}{\mu} \lambda_{\alpha}$

$\lambda_{0}=$ The natural length of the cable connecting the two satellites.

Here dashes denotes differentiations w.r.to true anomaly $v$.

The condition of constraint is given by,

$$
x^{2}+y^{2}+z^{2} \leq \frac{\lambda_{0}^{2}}{\rho^{2}}
$$

For the circular orbit of the centre of mass of the system, we must have $e=0$ and $\rho=\frac{1}{1+e \cos v}=1$ and $\rho^{1}=0$.

Putting $\rho=1$ and $\mathrm{i}=0$ (For equatorial orbit) in (1), we get the equations of motion for the system in three dimensional case as

$$
\begin{aligned}
& x^{\prime \prime}-2 y^{\prime}-(3+4 B) x+A \Psi_{1} \cos \in \cos (v-\alpha)=-\overline{\lambda_{\alpha}}\left[1-\frac{l_{0}}{r}\right] x-C \\
& y^{\prime \prime}+2 x^{\prime}+B y-A \Psi_{1} \cos \in \sin (v-\alpha)=-\overline{\lambda_{\alpha}}\left[1-\frac{l_{0}}{r}\right] y \\
& \text { and } z^{\prime \prime}+(1+B) z-A \Psi_{1} \sin \in=-\overline{\lambda_{\alpha}}\left[1-\frac{l_{0}}{r}\right] z
\end{aligned}
$$

Where, $r=\sqrt{x^{2}+y^{2}+z^{2}}$

The condition of constraint given by (2) takes the form

$$
x^{2}+y^{2}+z^{2} \leq \lambda_{0}^{2}
$$

We also assume that in case of circular orbit, the true anomaly $v$ for the elliptic orbit will be replaced by $\tau$ whose value is given below

$$
\tau=\omega_{0} t
$$

Where $\omega_{0}$ is the angular velocity of the centre of mass of the system in case of circular orbit and $t$ is the time.

Hence (3) can be written as

$$
\begin{aligned}
& x^{\prime \prime}-2 y^{\prime}-(3+4 B) x+A \Psi_{1} \cos \in \cos (\tau-\alpha)=-\bar{\lambda}_{\alpha}\left[1-\frac{\lambda_{0}}{r}\right] x-C \\
& y^{\prime \prime}+2 x^{\prime}+B y-A \Psi_{1} \cos \in \sin (\tau-\alpha)=-\overline{\lambda_{\alpha}}\left[1-\frac{\lambda_{0}}{r}\right] y \\
& \text { and } z^{\prime \prime}+(1+B) z-A \Psi_{1} \sin \in=-\bar{\lambda}_{\alpha}\left[1-\frac{\lambda_{0}}{r}\right] z
\end{aligned}
$$


If in (4), the inequality sign holds. Then the free motion of the system will take place otherwise motion will be constrained. Thus, we have three types of motion.

(i) Free motion (in case of loose string, $\overline{\lambda_{\alpha}}=0$ )

(ii) Constrained motion (in case of tight string, $\bar{\lambda}_{\alpha} \neq 0$ )

(iii) Evolutional motion (combination of free and constrained motion)

To find the Jacobian integral of the problem, we average the periodic terms in (6) as follows:$\frac{1}{2 \pi}\left[\int_{-\theta}^{\theta} A \psi_{1} \cos \in \cos (\tau-\alpha) d \tau+\int_{\Psi_{1}=0}^{2 \pi-\theta} A \psi_{1} \cos \in \cos (\tau-\alpha) d \tau\right]=\frac{-A \cos \in \cos \alpha \sin \theta}{\pi}$ and $\frac{1}{2 \pi}\left[\int_{-\theta}^{\theta} A \psi_{1} \cos \in \sin (\tau-\alpha) d \tau+\int_{\Psi_{1}=0}^{2 \pi-\theta} A \psi_{1} \cos \in \sin (\tau-\alpha) d \tau\right]=\frac{A \cos \in \operatorname{Sin} \alpha \sin \theta}{\pi}$

Where $\theta$ is taken to be constant.

Thus, the equations of motion given by (6) of the system are being described by using average values given in (7) in the form:-

$$
\begin{aligned}
& x^{\prime \prime}-2 y^{\prime}-(3+4 B) x-\frac{A \cos \in \cos \alpha \sin \theta}{\pi}=-\bar{\lambda}_{\alpha}\left[1-\frac{l_{0}}{r}\right] x-C \\
& y^{\prime \prime}+2 x^{\prime}+B y-\frac{A \cos \in \operatorname{Sin} \alpha \sin \theta}{\pi}=-\overline{\lambda_{\alpha}}\left[1-\frac{l_{0}}{r}\right] y \\
& \text { and } z^{\prime \prime}+(1+B) z-A \sin \in=-\overline{\lambda_{\alpha}}\left[1-\frac{l_{0}}{r}\right] z
\end{aligned}
$$

We see that the equations of motion given by (8) do not contain time texplicitly. Hence the................. (8) explicitly. Hence there must exist a Jacobian integral of the problem.

Multiplying the three equations of $(8)$ by $2 \mathrm{X}^{\prime}, 2 \mathrm{Y}^{\prime}$ 'and $2 \mathrm{z}^{\prime}$ respectively and adding then integrating, we get the jacobian integral in the form: $x^{\prime 2}+y^{\prime 2}+z^{\prime 2}-(3+4 B) x^{2}+B y^{2}+(1+B) z^{2}-\frac{1}{\pi} 2 A x \cos \in \cos \alpha \sin \theta$

$$
\frac{2 A y \cos \in \sin \alpha \sin \theta}{\pi}-2 A z \sin \in+\bar{\lambda}_{\alpha}\left[x^{2}+y^{2}+z^{2}-2 l_{0} \sqrt{x^{2}+y^{2}+z^{2}}\right]+2 C X=h
$$

Where $h$ is the constant of integration.

Equation (9) can be written as

$$
\begin{aligned}
& x^{\prime 2}+y^{\prime 2}+z^{\prime 2}=(3+4 B) x^{2}-B y^{2}-(1+B) z^{2}+\frac{1}{\pi} 2 A x \cos \in \cos \alpha \sin \theta \\
& +\frac{2 A y \cos \in \sin \alpha \sin \theta}{\pi}+2 A z \sin \in+\bar{\lambda}_{\alpha}\left[x^{2}+y^{2}+z^{2}-2 l_{0} \sqrt{x^{2}+y^{2}+z^{2}}\right]-2 C X+h
\end{aligned}
$$

Thus, the centre of zero velocity can be obtained in the form

$$
\begin{aligned}
& (3+4 B) x^{2}-B y^{2}-(1+B) z^{2}+\frac{1}{\pi} 2 A x \cos \in \cos \alpha \sin \theta+\frac{1}{\pi} 2 A y \cos \in \sin \alpha \sin \theta \\
& +2 A z \sin \in-\overline{\lambda_{\alpha}}\left[x^{2}+y^{2}+z^{2}-2 l_{0} \sqrt{x^{2}+y^{2}+z^{2}}\right]-2 C X+h=0 \\
& r=\sqrt{x^{2}+y^{2}+z^{2}}
\end{aligned}
$$


Hence, we conclude that the satellite of mass $m_{1}$ will move inside the boundaries of different curves of zero velocity represented by (11) of 10) for different values of Jacobian constant $h$.

\section{Equilibrium point of the problem}

We have obtained the set of equations given by (8) for the motion of the system in rotating frame of reference. It has been supposed that the system is moving with effective constraints and considering cable connecting the two satellites to be tight.

The equilibrium positions of the system are given by the constant values of the coordinates in the rotating frame of reference.

Now, let $x=x_{0}, y=y_{0}$ and $\mathrm{z}=\mathrm{z}_{0}$ give the equilibrium position

Where $x_{0} \quad y_{0}$ and $z_{0}$ are constant. and

Hence $x^{\prime}=0=x^{\prime \prime} \quad y^{\prime}=0=y^{\prime \prime}$ and $z^{\prime}=0=z^{\prime \prime}$

Thus, equations given by (8) take the form:

$$
\begin{array}{r}
-(3+4 B) x_{0}-\frac{A}{\pi} \cos \in \cos \alpha \sin \theta=-\overline{\lambda_{\alpha}}\left[1-\frac{\lambda_{0}}{r_{0}}\right] x_{0} \\
B y_{0}-\frac{A}{\pi} \cos \in \operatorname{Sin} \alpha \sin \theta=-\overline{\lambda_{\alpha}}\left[1-\frac{\lambda_{0}}{r_{0}}\right] y \\
(1+B)_{Z_{0}}-A \sin \in=-\overline{\lambda_{\alpha}}\left[1-\frac{l_{0}}{r}\right] Z_{0}
\end{array}
$$

Where, $r_{0}=\sqrt{x_{0}^{2}+y_{0}^{2}+z_{0}^{2}}$

From (12) it follows that it is very difficult to get the solution in its present form. Hence we are compelled to make the following assumptions; $\alpha=0$ and $\in=0$ in (12) and hence

We get

$$
\begin{array}{r}
-(3+4 B) x_{0}-\frac{A}{\pi} \sin \theta=-\bar{\lambda}_{\alpha}\left[1-\frac{\ell_{0}}{r_{0}}\right] x_{0}-C \\
B y_{0}=-\bar{\lambda}_{\alpha}\left[1-\frac{\ell_{0}}{r_{0}}\right] y_{0} \\
(1+\mathrm{B}) \mathrm{z}_{0}=-\bar{\lambda}_{\alpha}\left[1-\frac{\ell_{0}}{r_{0}}\right] z_{0}
\end{array}
$$

From (13), we get the equilibrium point as

$$
\left[\frac{\overline{\lambda_{\alpha}} \ell_{0} \pi+A \sin \theta-C \pi}{\pi\left(\overline{\lambda_{\alpha}}-3-4 B\right)}, 0,0\right]
$$

It can be easily seen that the equilibrium point given by (14) gives us a meaningful value of Hooke's modules of elasticity.

\section{Stability of the equilibrium point of the system}

We shall examine the stability of the equilibrium point of the system given by (14) in the sense of Liapunov's. For this,

Let, $x=a=\frac{\overline{\lambda_{\alpha}} \pi \ell_{0}+A \sin \theta-C \pi}{\pi\left(\overline{\lambda_{\alpha}}-3-4 B\right)}, \mathrm{y}=0, \mathrm{z}=0$ 
Let us assume that there are small variations in the coordinates at the given equilibrium position. Let $\eta_{1}, \eta_{2}$ and $\eta_{3}$ denote small variations in the coordinates for this position of equilibrium. Then we have

$$
\begin{aligned}
& x=a+\eta_{1} \quad y=0+\eta_{2} \quad z=0+\eta_{3} \\
& \therefore \quad x^{\prime}=\eta_{1}^{\prime}, \quad y^{\prime}=\eta_{2}^{\prime} \quad z^{\prime}=\eta_{3}^{\prime} \\
& x^{\prime \prime}=\eta_{1}^{\prime}, \quad y^{\prime \prime}=\eta_{2}^{\prime \prime} \quad z^{\prime \prime}=\eta_{3}^{\prime \prime}
\end{aligned}
$$

Using (15) in (8), we get the variational equations of motion for the system in the form :

$$
\begin{gathered}
\eta_{1}^{\prime \prime}-2 \eta_{2}^{\prime}-(3+4 B)\left(a+\eta_{1}\right)-\frac{A \sin \theta}{\pi}=-\bar{\lambda}_{\alpha}\left[1-\frac{\ell_{0}}{r}\right]\left(a+\eta_{1}\right)-C \\
\eta_{2}^{\prime \prime}+2 \eta_{1}^{\prime}+B \eta_{2}=-\bar{\lambda}_{\alpha}\left[1-\frac{\ell_{0}}{r}\right] \eta_{2} \\
\eta_{3}^{\prime \prime}+(1+B) \eta_{3}=-\bar{\lambda}_{\alpha}\left[1-\frac{\ell_{0}}{r}\right] \eta_{3}
\end{gathered}
$$

Where,$r^{2}=\left(a+\eta_{1}\right)^{2}+\eta_{2}^{2}+\eta_{3}^{2}$

Here $\epsilon=0$ and $\alpha=0$ have been taken into considerations as a particular case for our further study.

Multiplying the three equations of (16) by $2\left(a+\eta_{1}\right), 2 \eta_{2}^{\prime}$ and $2 \eta_{3}^{\prime}$ respectively and then adding them together and integrating, we get Jacobian integral in the form :

$$
\begin{gathered}
\eta_{1}^{\prime 2}+\eta_{2}^{\prime 2}+\eta_{3}^{\prime 2}-(3+4 B)\left(a+\eta_{1}\right)^{2}+(1+B) \eta_{3}^{2}-\frac{2 A \eta_{1} \sin \theta}{\pi} \\
+\bar{\lambda}_{\alpha}\left[\left(a+\eta_{1}\right)^{2}+\eta_{2}^{2}+\eta_{3}^{2}\right]-2 \bar{\lambda}_{\alpha} \ell_{0}\left[\left(a+\eta_{1}\right)^{2}+\eta_{2}^{2}+\eta_{3}^{2}\right]^{\frac{1}{2}}+2 C\left(a+\eta_{1}\right)=h
\end{gathered}
$$

Where $h$ is the constant of integration.

To examine the stability in the sense of Liafunov's, we take Jacobi's integral given by (17) as Liapunov's function $v\left(\eta_{1}^{\prime}, \eta_{2}^{\prime}, \eta_{3}^{\prime}, \eta_{1}, \eta_{2}, \eta_{2}\right)$ and is obtained by expanding the terms of (17) as

$$
\begin{gathered}
v\left(\eta_{1}^{\prime}, \eta_{2}^{\prime}, \eta_{3}^{\prime}, \eta_{1}, \eta_{2}, \eta_{3}\right)=\eta_{1}^{\prime 2}+\eta_{2}^{\prime 2}+\eta_{3}^{\prime 2} \\
+\eta_{1}^{2}\left[\overline{\lambda_{\alpha}}-3-4 B-\frac{\overline{\lambda_{\alpha}} \ell_{0}}{a}\right]+\eta_{2}^{2}\left[\overline{\lambda_{\alpha}}-\frac{\overline{\lambda_{\alpha}} \ell_{0}}{a}\right]+\eta_{3}^{2}\left[1+B+\overline{\lambda_{\alpha}}-\frac{\overline{\lambda_{\alpha}} \ell_{0}}{a}\right] \\
+\eta_{1}\left[-2(3+4 B) a+2 C-\frac{2 A \sin \theta}{\pi}+2 a \overline{\lambda_{\alpha}}-2 \overline{\lambda_{\alpha}} \ell_{0}\right] \\
+a\left[-(3+4 B) a+2 C+\overline{\lambda_{\alpha}} 2\left(a-\ell_{0}\right)\right]+0(3)
\end{gathered}
$$

Where $0(3)$ stand for the third and higher order terms in the small quantities $\eta_{1}, \eta_{2}$ and $\eta_{3}$.

By Liapunov's theorem on stability it follows that the only criterion for given equilibrium position $(\mathrm{a}, \mathrm{o}, \mathrm{o})$ to be stable is that $v$ defined by (18) must be positive definite and for this the following four conditions must be satisfied :

$$
\begin{aligned}
& \text { (i) }-2(3+4 B) a+2 C-\frac{2 A \sin \theta}{\pi}+2 \overline{\lambda_{\alpha}} a-2 \bar{\lambda}_{\alpha} \ell_{0}=0 \\
& \text { (ii) }\left(\overline{\lambda_{\alpha}}-3-4 B-\frac{\overline{\lambda_{\alpha}} \ell_{0}}{a}\right)>0 \\
& \text { (iii) } \overline{\lambda_{\alpha}}-\frac{\overline{\lambda_{\alpha}} \ell_{0}}{a}>0
\end{aligned}
$$


(iv)

$$
1+B+\overline{\lambda_{\alpha}}-\frac{\overline{\lambda_{\alpha}} \ell_{0}}{a}>0
$$

Now the different conditions for the stability of the equilibrium may be examined separately to have a clear picture of the stability of the system at the obtain equilibrium position.

\section{Condition (I):}

The first condition can be written as on putting the value of a as

$$
\begin{aligned}
& \text { L.H.S. }=-2(3+4 B)\left[\frac{\overline{\lambda_{\alpha}} \pi \ell_{0}+A \sin \theta-C \pi}{\pi\left(\overline{\lambda_{\alpha}}-3-4 B\right)}\right]+2 C-\frac{2 A \sin \theta}{\pi}+2 \overline{\lambda_{\alpha}} \frac{\overline{\lambda_{\alpha}} \pi \ell_{0}+A \sin \theta-C \pi}{\pi\left(\overline{\lambda_{\alpha}}-3-4 B\right)}-2 \bar{\lambda}_{\alpha} \ell_{0} \\
& =\frac{2\left(\overline{\lambda_{\alpha}} \pi \ell_{0}+A \sin \theta-C \pi\right)\left[\overline{\lambda_{\alpha}}-3-4 B\right]+2 C}{\pi\left(\overline{\lambda_{\alpha}}-3-4 B\right)}-\frac{2 A \sin \theta}{\pi}-2 \bar{\lambda}_{\alpha} \ell_{0} \\
& =2 \bar{\lambda}_{\alpha} \ell_{0}+\frac{2 A \sin \theta}{\pi}-\frac{2 A \sin \theta}{\pi}-2 \bar{\lambda}_{\alpha} \ell_{0}+2 C-2 C=0
\end{aligned}
$$

Hence we see that the condition (i) is identically satisfied.

\section{Condition (II):}

Before analysing this condition, the values of a in the equilibrium position $(a, 0,0)$ is taken for consideration, then we have in the equilibrium position:

$$
\begin{aligned}
& a=\frac{\overline{\lambda_{\alpha}} \pi \ell_{0}+A \sin \theta-C \pi}{\pi\left(\overline{\lambda_{\alpha}}-3-4 B\right)}>0 \\
& \Rightarrow \overline{\lambda_{\alpha}} \pi \ell_{0}+A \sin \theta-C \pi>0 \text { and } \quad \pi\left(\overline{\lambda_{\alpha}}-3-4 B\right)>0 \\
& \text { But } \overline{\lambda_{\alpha}} \pi \ell_{0}+A \sin \theta-C \pi>0 \quad \text { as } \quad \overline{\lambda_{\alpha}}>0, \quad \pi>0, \quad \ell_{0}>0, \mathrm{~A}>0 \text { and } 0<\theta<\pi / 2 \text { and }
\end{aligned}
$$

$\mathrm{C}$ is small. Hence, $\overline{\lambda_{\alpha}}-3-4 B>$

$$
\begin{aligned}
\mid\left(\overline{\lambda_{\alpha}}-3-4 B\right) & \left.-\frac{\overline{\lambda_{\alpha}} \pi \ell_{0}\left(\overline{\lambda_{\alpha}}-3-4 B\right)}{\overline{\lambda_{\alpha}} \pi \ell_{0}+A \sin \theta-C \pi}\right\rfloor \\
& =\quad\left(\overline{\lambda_{\alpha}}-3-4 B\right) \frac{(A \sin \theta-C \pi)}{\overline{\lambda_{\alpha}} \pi \ell_{0}+A \sin \theta-C \pi}>0
\end{aligned}
$$

Using $\overline{\lambda_{\alpha}} \pi \ell_{0}+A \sin \theta-C \pi>0, \overline{\lambda_{\alpha}}-3-4 B>0$ and $\mathrm{C}$ is small

Hence 2 nd condition is identically satisfied.

\section{Condition (III):}

$$
\begin{aligned}
& \text { L.H.S of (iii) }=\overline{\lambda_{\alpha}}-\frac{\bar{\lambda}_{\alpha} \ell_{0}}{a}=\overline{\lambda_{\alpha}}\left(1-\frac{\ell_{0}}{a}\right)=\overline{\lambda_{\alpha}}\left[1-\frac{\ell_{0}\left(\pi \bar{\lambda}_{\alpha}-3 \pi-4 B \pi\right)}{\bar{\lambda}_{\alpha} \ell_{0} \pi+A \sin \theta-C \pi}\right] \\
& =\overline{\lambda_{\alpha}}\left[\frac{\overline{\lambda_{\alpha}} \pi \ell_{0}-\pi \ell_{0} \bar{\lambda}_{\alpha}+\pi \ell_{0}+A \sin \theta+(3+4 B)-C \pi}{\bar{\lambda}_{\alpha} \pi \ell_{0}+A \sin \theta-C \pi}\right] \\
& =\overline{\lambda_{\alpha}}\left[\frac{A \sin \theta+(3+4 B) \pi \ell_{0}-C \pi}{\bar{\lambda}_{\alpha} \pi \ell_{0}+A \sin \theta-C \pi}\right]>0 \\
& \text { Using } \frac{\bar{\lambda}_{\alpha} \pi \ell_{0}+A \sin \theta-C \pi>0, \overline{\lambda_{\alpha}}>0 \text { and (3+4B) } \ell_{0} \pi>0}{}
\end{aligned}
$$

Hence third condition is also satisfied.

\section{Condition (IV):}


L.H.S of (iii) $=1+B+\overline{\lambda_{\alpha}}-\frac{\overline{\lambda_{\alpha}} \ell_{0}}{a}=1+B+\overline{\lambda_{\alpha}}\left(1-\frac{\ell_{0}}{a}\right)>0$

Using condition (III) and $\mathrm{B}>0$.

Hence fourth condition is also identically satisfied.

Thus, we see that all the four conditions of (19) for stability of the equilibrium position (a, 0,0$)$ are identically satisfied.

Thus, we conclude that the equilibrium point $(a, 0,0)$ given by (14) of the system is stable in the sense of Liapunov

\section{Conclusion:}

We conclude that the equilibrium point $(a, 0,0)$ given by (14) of the system is stable in the sense of Liapunov

\section{Reference:}

[1]. Beletsky, V. V.: About the Relative Motion of Two Connected Bodies in orbit. Kosmicheskiye Issoledovania, vol. 7, No. 6, pp. 827 840, 1969 (Russian).

[2]. Thakur ; H.K. : The motion of a system of two satellites connected by extensible cable ;Ph.D. thesis, submitted to B. R. A. Bihar University, Muzaffarpur, 1975

[3]. Singh, R. B.: Three Dimensional motion of system a two cable-connected satellite in orbit. Astronautica, acta, vol. 18, pp. 301 308, 1973

[4]. Singh, A. K. P. : Effect of Earth's Shadow on the motion of a system of two Satellites connected by extensible cable under the influence of solar radiation pressure, Ph. D. Thesis Submitted to B. R. A. Bihar University, Muzaffarpur, 1990.

[5] V. Kumar and N. Kumari, Stability of Equilibrium point of the centre of mass of an extensible cable connected satellites system in case of circular orbit in three dimensional, IJSER, Vol-4,Issue 9, (2013), 1802-1808 